\title{
Continuity, Completeness and the Definition of Weak Preferences
}

\author{
Edi Karni* \\ Johns Hopkins University
}

May 30, 2011

\begin{abstract}
This note explores the connections between continuity and completeness under alternative conceptions of preference relations. For non-trivial preorders, it shows that, unlike the standard definitions, the weak preference relation defined in Galaabataar and Karni (2010) allows for incomplete preferences while maintaining all the continuity properties of complete preference relations. It also makes it possible to distinguish indifference between alternatives from non-comparability of alternatives. If the preference relations are complete, this definition agrees with the customary definitions.
\end{abstract}

Keywords: Preference relations, continuity, Archimedean axiom, mixture continuity, complete preorders, incomplete preferences

JEL classification numbers: D01, D81

*I am grateful to Juan Dubra, Tsogbadral Galaabataar, Zvi Safra, two anonymous referees and an associate editor for their useful comments and suggestions. 


\section{Introduction}

Prompted by recent works of Galaabaatar and Karni (2010) and Dubra (2011), this note explores the connections between the properties of completeness and continuity under alternative definitions of preference relations.

The study of the connections between continuity and completeness was pioneered by Schmeidler (1971), who proved that a non-trivial preorder on a connected topological space is continuous (that is, the upper and lower contour sets are closed and the upper and lower contour sets corresponding to the asymmetric part of the preorder are open) only if it is complete. Building upon Schmeidler's result, Dubra (2011) showed that if a preorder on the set of lotteries (that is, probability distributions on a finite set of prizes) is non-trivial and satisfies the independence axiom of expected utility theory, then any two of the following three axioms implied the third, Archimedean, mixture continuity and completeness. Consequently, a non-trivial, partial, preorder satisfying the independence axiom must fail to satisfy one of the continuity axioms.

Galaabaatar and Karni (2010) showed that a non-trivial preorder on the set of lotteries satisfying the independence and Archimedean axioms can be incomplete. Moreover, it is shown below that such preorder also satisfies mixture continuity.

The key to understanding why these, seemingly contradictory, results are nevertheless all valid is a subtle difference in the definitions of the preference relations under consideration. In particular, following the traditional approach, Schmeidler (1971) and Dubra (2011), take the weak preference relation as primitive and define the corresponding strict preference relation as its asymmetric part. ${ }^{1}$ By contrast, Galaabaatar and Karni (2010) take the strict preference relation as primitive and define one alternative to be weakly preferred over another if every alternative that is strictly preferred over the former is strictly preferred over the latter. In this note, I show that this difference between the two definitions accounts for the distinct implications indicated by the aforementioned results.

An alternative definition of a weak preference relation that, like Galaabaatar and Karni (2010), takes strict preference relation for primitive, defines the weak preference relation as the negation of the strict preference relation in

\footnotetext{
${ }^{1}$ Dubra et al. (2004) invoke the same approach.
} 
the inverse sense. I show, in the next section, that the two definitions of weak preference relations induced by the same strict preference relation are complete preorders if and only if they are equivalent. However, only the definition of Galaabaatar and Karni (2010) makes it possible to separate indifference from incomparability.

\section{Alternative Concepts of Induced Weak Pref- erences}

We are concerned with the definitions of preference relations on an abstract choice set, $T$, where $T$ is a connected topological space. To begin with, consider the approach that invokes, as primitive, a non-trivial strict preference relation, $\succ$ on $T$, (that is $\succ$ is non-empty, transitive, and irreflexive binary relation) and use it to define weak preference relations on $T$.

\subsection{Two definitions of induced weak preference rela- tions}

Let $\succ$ on $T$ be a strict preference relation. Define a weak preference relation, $\succcurlyeq$ on $T$, as the negation of the strict preference relation in the inverse sense. Formally,

Definition 1: For all $p, q \in T, p \succcurlyeq q$ if $\neg(q \succ p)$.

Since $\succ$ is transitive and irreflexive then it is asymmetric and, consequently, $\succcurlyeq$ is complete (that is, for all $p, q \in T, p \succcurlyeq q$ or $q \succcurlyeq p$ ), but not necessarily transitive. If $\succ$ is also negatively transitive (that is, $\neg(q \succ p)$ and $\neg(p \succ r)$ then $\neg(q \succ r))$ then $\succcurlyeq$ is transitive. Note, however, that if $\neg(p \succ q)$ and $\neg(q \succ p)$ then $p \succcurlyeq q$ and $q \succcurlyeq p$. If we define an equivalence relation, $\sim$ on $T$, by $p \sim q$ if $p \succcurlyeq q$ and $q \succcurlyeq p$, that is, the absence of strict preference, then $p \sim q$ may be interpreted to mean that $p$ is indifferent to

$q$ or that $p$ and $q$ are non-comparable. Definition 1 does not allow us to distinguish between these two interpretations. 
An alternative definition of weak preference relation, $\hat{\succcurlyeq}$ on $T$, introduced in Galaabaatar and Karni (2010), is as follows: ${ }^{2}$

Definition 2: For all $p, q \in T, p \hat{\succcurlyeq} q$ if $r \succ p$ implies $r \succ q$, for all $r \in T$.

Notice that, according to Definition $1, \succ$ is the asymmetric part of $\succcurlyeq$ (that is, $p \succcurlyeq q$ and $\neg(q \succcurlyeq p)$ imply $p \succ q)$. By contrast, according to Definition $2, p \succcurlyeq q$ and $\neg(q \succcurlyeq p)$ do not imply that $p \succ q$. Thus, $\succ$ is not the asymmetric part of $\hat{\succcurlyeq}$. Thus, in general, the two concepts of weak preference relations are distinct, even though they are induced by the same primitive strict preference relation. More importantly, unlike Definition 1, the induced preference relation $\hat{\succcurlyeq}$ is transitive but not necessarily complete. ${ }^{3}$ Moreover, if we define $p \hat{\sim} q$ as $p \hat{\succcurlyeq} q$ and $q \hat{\succcurlyeq} p$ then $\hat{\sim}$ is an indifference relation, while the binary relation $p \bowtie q$ defined by $\neg(p \hat{\succcurlyeq} q)$ and $\neg(q \hat{\succcurlyeq} p)$ has the interpretation that $p$ and $q$ are not comparable. Hence, unlike Definition 1, Definition 2, makes it possible to distinguish indifference from non-comparability. ${ }^{4}$

\subsection{On the equivalence of the two definitions}

I show next that the two definitions are equivalent if and only if $\hat{\succcurlyeq}$ is complete and $\succcurlyeq$ is transitive. I assume throughout that $\succ$ is non-trivial, transitive and irreflexive binary relation on $T$.

Proposition: Let $\succcurlyeq$ and $\hat{\succcurlyeq}$ be the binary relations on $T$ given in Definitions 1 and 2, respectively. Then, $\succcurlyeq=\succcurlyeq$ if and only if $\succ$ is negatively transitive and $\hat{\succcurlyeq}$ is complete.

Proof. Suppose that $\succcurlyeq=\hat{\succcurlyeq}$ and that $\hat{\succcurlyeq}$ is not complete. Then $\succcurlyeq$ is not complete. Thus, $\succ$ is not asymmetric. Hence, there exist $p, q \in T$ such that $p \succ q$ and $q \succ p$. By transitivity of $\succ, p \succ p$, which contradicts the irreflexivity. Thus, $\succcurlyeq$ is complete.

\footnotetext{
${ }^{2}$ Chateauneuf (1987) applies a similar idea, using a pseudo-transitive weak preference relation to induce other weak preference relations that are shown to be complete.

${ }^{3}$ Notice that, unlike $\succcurlyeq$, the transitivity of $\hat{\succcurlyeq}$ does not require that $\succ$ be negatively transitive.

${ }^{4} \mathrm{~A}$ preference relation is said to display IP-transitivity if $p$ is indifferent to $q$ and $q \succ r$ then $p \succ r$. In general, the preference relation $\hat{\succcurlyeq}$ displays IP-transitivity.
} 
Suppose that $\succcurlyeq=\hat{\succcurlyeq}$ and $\succ$ is not negatively transitive. Then there exist $p, q, r \in T$ such that $\neg(p \succ q), \neg(q \succ r)$ and "not $\neg(p \succ r)$ ". Hence, by Definition $1, q \succcurlyeq p, r \succcurlyeq q$ and $p \succ r$. Hence, $\succcurlyeq$ in not transitive. But $\hat{\succcurlyeq}$ is transitive. Hence, $\neg(\succcurlyeq=\hat{\succcurlyeq})$. A contradiction.

Suppose that $\succ$ is negatively transitive and $\hat{\succcurlyeq}$ is complete. Then, by negative transitivity of $\succ, \succcurlyeq$ is transitive. If $p \succcurlyeq q$ then, by transitivity of $\succ$ and $\succcurlyeq, r \succ p$ implies $r \succ q$, for all $r \in T$. Thus, by Definition $2, p \succcurlyeq q$. Hence, $\succcurlyeq \subset \hat{\succcurlyeq}$. Let $p \hat{\succcurlyeq} q$. If $\neg(p \succcurlyeq q)$ then, by Definition $1, q \succ p$. Hence, $p \hat{\succcurlyeq} q$ implies $q \succ q$, contradicting the irreflexivity of $\succ$. Hence, $\succcurlyeq \supset \succcurlyeq$. Thus, $\succcurlyeq=\succcurlyeq$.

The weak preference relation in Definition 2 may be incomplete, in which case it distinguishes indifference from incomparability. By contrast, the weak preference relation in Definition 1 is complete and too coarse to permit such a distinction.

\section{Continuity and Completeness}

The standard approach in decision theory is to take, as primitive a weak preference relation, $\succcurlyeq$, defined to be non-trivial preorder on $T$, and define the strict preference relation, $\succ$, to be its asymmetric part. Formally, let $\succcurlyeq$ be a non-trivial, transitive and reflexive binary relation on $T$,

Definition 3: For all $p, q \in T, p \succ q$ if $p \succcurlyeq q$ and $\neg(q \succcurlyeq p)$.

I show next that if $\succcurlyeq$ is incomplete, then certain continuity properties of complete preference relations are lost. By contrast, the weak preference relation in Definition 2 may be incomplete while preserving the continuity properties of complete preference relations. Notice, however, that these results do not contradict the theorems of Schmeidler (1971) and Dubra (2011), rather they demonstrate the important and subtle role played by Definition 3 in their respective frameworks.

\subsection{Schmeidler's theorem revisited}

Schmeidler (1971) proved the following theorem:

Schmeidler's Theorem: Let $T$ be a connected topological space. Let $\succcurlyeq$ be a binary relation on $T$ and suppose that $\succ$ is given as in Definition 
3. If $\succcurlyeq$ is transitive, non-trivial and such that for each $q \in T$, the sets $\{p \in T \mid p \succ q\}$ and $\{p \in T \mid q \succ p\}$ are open and the sets $\{p \in T \mid p \succcurlyeq q\}$ and $\{p \in T \mid q \succcurlyeq p\}$ are closed, then it is complete.

Schmeidler's proof includes the argument that if $p \succcurlyeq q$ and not $p \succ q$ then $q \succcurlyeq p$. Note that, according to Definition 2 it is possible that $p \succcurlyeq q$ and not $p \succ q$ and yet, not $q \hat{\succcurlyeq} p$. Thus, this argument does not apply if the weak preference relation $\hat{\succcurlyeq}$ is derived from $\succ$ as in Definition 2. Moreover, according to Definition 2, it is possible that $p \succ q$ and

$$
\{r \in T \mid r \succ q\} \cup\{r \in T \mid p \succ r\}
$$

is a proper subset of $T$. This is illustrated by the following example. Let $T$ be the simplex in $\mathbb{R}^{3}$. Let $X=\{\$ 0, \$ 500, \$ 1000\}$. Denote by $\delta_{x}$, the degenerate lottery that assigns to $x$ the unit probability mass. Suppose that $\delta_{\$ 1000} \succ$ $p \succ \delta_{\$ 0}$, for all $p \in T-\left\{\delta_{\$ 0}, \delta_{\$ 1000}\right\}$, and

$$
\mathbb{U}:=\{u: X \rightarrow \mathbb{R} \mid u(\$ 1000)=1, u(\$ 0)=0, u(\$ 500)=\alpha, \alpha \in[0.5,0.75]\} .
$$

Define binary relations $\succ$ and $\hat{\succcurlyeq}$ on $T$ as follows:

$$
p \succ q \Leftrightarrow \Sigma_{x \in X} u(x) p(x)>\Sigma_{x \in X} u(x) q(x), \forall u \in \mathbb{U},
$$

and

$p \hat{\succcurlyeq} q \Leftrightarrow \Sigma_{x \in X} u(x) p(x) \geq \Sigma_{x \in X} u(x) q(x), \forall u \in \mathbb{U}$ with equality for some $u \in \mathbb{U}$.

Then, the preference relation $\hat{\succcurlyeq}$ is transitive, nontrivial, satisfies the topological continuity properties in Schmeidler's theorem and the independence axiom of expected utility theory. However, the preference relation is incomplete. This is depicted in Figure 1 below. The lines through $p$ and $q$ represent the indifference curves corresponding to utility functions in $\mathbb{U}$ given by $u(\$ 500)=0.5$ and $u(\$ 500)=0.75$. As the diagram clearly shows, the areas marked by $K$ are points in $T-\{r \in T \mid r \succ q\} \cup\{r \in T \mid p \succ r\}$.

Place Figure 1 here

It is important to note that the continuity properties in Schmeidler's theorem are satisfied since $\hat{\succcurlyeq}$ satisfies the closedness conditions and $\succ$ salsifies the openness conditions. However, the strict preference relation $\succ$ is not the asymmetric part of $\succcurlyeq$. Hence, the continuity conditions in Schmeidler's theorem do not imply completeness. 


\subsection{Dubra's theorem revisited}

Let $X$ be a finite set of outcomes and denote by $\Delta(X)$ the set of probability distributions on $X$. Define the mixture operation on $\Delta(X)$ as follows: For all $p, q \in \Delta(X)$ and $\alpha \in[0,1],(\alpha p+(1-\alpha) q)(x)=\alpha p(x)+(1-\alpha) q(x)$, for all $x \in X$. Then $\Delta(X)$ is a convex subset of a linear space. Moreover, endowing the space with the $\mathbb{R}^{n}$ topology, it is a connected topological space.

Let $\succcurlyeq$ be a preorder on $\Delta(X)$ and suppose that its asymmetric part, $\succ$, is given by Definition 3. Assume throughout that $\succ$ is non-trivial and satisfies the independence axiom below.

Independence - For all $p, q, r \in C$ and $\alpha \in(0,1], p \succ q$ if and only if $\alpha p+(1-\alpha) r \succ \alpha q+(1-\alpha) r$.

The induced binary relation $\succcurlyeq$ may be continuous. The following axioms were used to characterize the continuity of $\succcurlyeq$ :

Archimedean - For all $p, q, r \in \Delta(X)$, if $p \succ q$ and $q \succ r$ then there are $\alpha, \beta \in(0,1)$ such that $\beta p+(1-\beta) r \succ q$ and $q \succ \alpha p+(1-\alpha) r$.

Mixture continuity - For all $p, q, r \in \Delta(X)$ the sets $\{\alpha \in[0,1] \mid \alpha p+$ $(1-\alpha) q \succcurlyeq r\}$ and $\{\alpha \in[0,1] \mid r \succcurlyeq \alpha p+(1-\alpha) q\}$ are closed.

Dubra (2011) proved the following result:

Dubra's Theorem: Let $\succcurlyeq$ be a transitive, reflexive, non-trivial binary relation on $\Delta(X)$ satisfying independence, and suppose that $\succ$ is given as in Definition 3. Then, if $\succcurlyeq$ satisfies any two of the following axioms, Archimedean, mixture continuity and completeness, then it satisfies the third.

Thus, a partial preference relation cannot satisfy both the Archimedean and mixture continuity axioms.

Galaabaatar and Karni (2010) showed that there exist nontrivial, transitive and irreflexive, strict preference relations on $\Delta(X)$ satisfying the independence and Archimedean axioms that induces, under Definition 2, partial 
weak preference relation. Moreover, the example above shows that the binary relations in their model also satisfy mixture continuity. ${ }^{5}$ Thus, strict preference relations and the derived weak preference relations characterized in Definition 2, may be non-trivial and satisfy the independence, both continuity axioms, and still be incomplete.

It is worth emphasizing again that, even though the strict preference relation $\succ$ satisfies the Archimedean and Independence axioms, Dubra's theorem is not contradicted by this argument since $\succ$ is not the asymmetric part of $\hat{\succcurlyeq}$, which satisfies Mixture Monotonicity.

\footnotetext{
${ }^{5}$ Take a sequence of $\left\{\alpha_{n}\right\}$ converging to $\alpha^{*}$ such that $\alpha_{n} p+\left(1-\alpha_{n}\right) q \succcurlyeq r$, for all $n=1,2, \ldots$. Then,

$\alpha_{n} \Sigma_{x \in X} u(x) p(x)+\left(1-\alpha_{n}\right) \Sigma_{x \in X} u(x) q(x) \geq \Sigma_{x \in X} u(x) r(x)$, for all $u \in \mathbb{U}$ and $n=1,2, \ldots$
}

Hence, in the limit,

$$
\alpha^{*} \Sigma_{x \in X} u(x) p(x)+\left(1-\alpha^{*}\right) \Sigma_{x \in X} u(x) q(x) \geq \Sigma_{x \in X} u(x) r(x) \text {, for all } u \in \mathbb{U} \text {. }
$$

Thus, $\alpha^{*} p+\left(1-\alpha^{*}\right) q \hat{\succcurlyeq}$. Hence, $\hat{\succcurlyeq}$ satisfies mixture continuity. 


\section{References}

[1] Chateauneuf, Alain (1987) "Continuous Representation of a Preference Relation on a Connected Topological Space," Journal of Mathematical Economics, 16, 139-146.

[2] Dubra, Juan (2011) "Continuity and Completeness under Risk," Mathematical Social Sciences, 61, 80-81.

[3] Dubra, Juan, Maccheroni, Fabio and Ok, Efe A. (2004) "Expected Utility Theory without the Completeness Axiom," Journal of Economic Theory, $115,118-133$.

[4] Galaabaatar, Tsogbadral and Karni Edi (2010) "Objective and Subjective Expected Utility with Incomplete Preferences," unpublished manuscript

[5] Schmeidler, David (1971) "A Condition for the Completeness of Partial Preference Relations," Econometrica, 39, 403. 